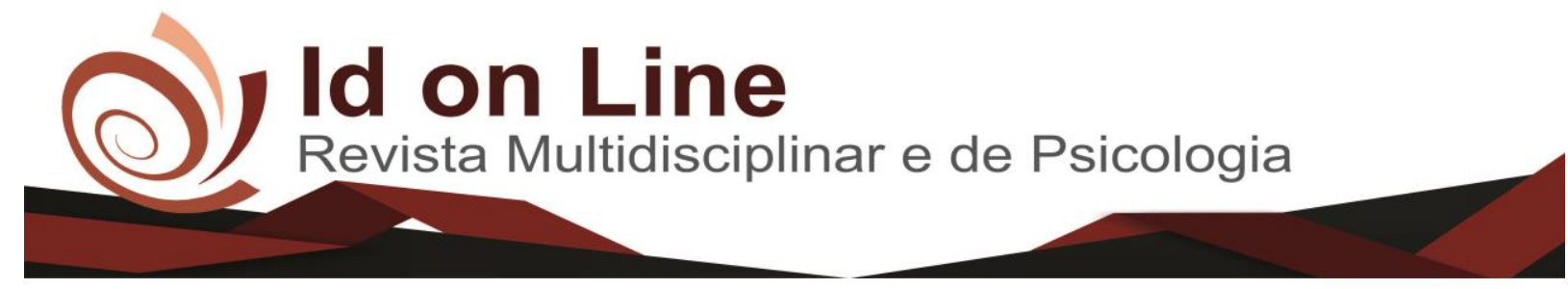

Artigo

\title{
Prevalência de Doenças Crônicas não Transmissíveis em Idosos de uma Cidade do Interior da Bahia
}

\author{
Sanmille Santos Santiago de Abreu ${ }^{l}$; Andreza Guimarães de Oliveira ${ }^{l}$; Maria Aparecida Silva Santos Macedo ${ }^{1}$ \\ Stênio Fernando Pimentel Duarte ${ }^{2}$; Luciana Araújo dos Reis ${ }^{3}$; Pollyanna Viana Lima ${ }^{4}$
}

\begin{abstract}
Resumo: Objetivo: Verificar a prevalência das Doenças Crônicas Não Transmissíveis em idosos de uma cidade do interior da Bahia. Métodos: A pesquisa se classifica como descritiva e exploratória, de natureza quantitativa que foi realizada com 173 idosos de um município do interior da Bahia Foram aplicados dois instrumentos de pesquisa: o Mini Exame do Estado Mental - MEEM e Ficha de doenças pré-existentes - critérios ABCDE. Resultados: Os participantes do estudo foram 173 idosos de 60 anos ou mais de ambos os sexos, 54,91\% dos idosos do sexo feminino e 45,09\% do sexo masculino. No que se refere à População Feminina, 1,15\% auto-referiram ser diagnosticado com a depressão, 15,02\% diabetes, 2,89 cardiopatia, 2,89 doença renal, 0,58\% câncer, 71,68\% hipertensão. Quanto à população masculina auto-referiram ser diagnosticado com diabetes 2,90\%, 1,73\% cardiopatia, 2,31 câncer e 27,16\% hipertensão. Conclusão: Constatou-se que o perfil de morbidades crônicas encontrados na população pesquisada é semelhante ao perfil em idosos no Brasil. Observa-se tendência crescente especialmente na Hipertensão Arterial Sistêmica, Diabetes Mellitus e câncer na população masculina. O estudo demonstrou ainda um quantitativo maior de doenças auto-referidas por mulheres.
\end{abstract}

Descritores: Idosos; Doenças crônicas; envelhecimento.

\section{Non-Communicable Chronic Diseases Prevalence in the Elderly of a City of the Interior of Bahia}

\begin{abstract}
Objective: To verify the prevalence of chronic noncommunicable diseases in the elderly in a city in the interior of Bahia. Methods: The research is classified as descriptive and exploratory, of a quantitative nature that was performed with 173 elderly people from a municipality in the interior of Bahia. Two research instruments were applied: the Mini Mental State Examination (MMSE) and the Pre- ABCDE criteria. RESULTS: The study participants were 173 elderly of 60 years and older of both sexes, $54.91 \%$ of the elderly female and $45.09 \%$ male. Regarding the Female Population, 1.15\% self-reported being diagnosed with depression, 15.02\% diabetes, 2.89 heart disease, 2.89 renal disease, $0.58 \%$ cancer, $71.68 \%$ hypertension. As for the male self-referenced being diagnosed with diabetes $2.90 \%, 1.73 \%$ heart disease, 2.31 cancer and $27.16 \%$ hypertension. Conclusion: It was verified that the profile of chronic morbidity found in the population studied is similar to the profile in the elderly in Brazil. There is a growing trend especially in Systemic Arterial Hypertension, Diabetes Mellitus and cancer in the male population. The study also demonstrated a larger number of self-reported diseases by women.
\end{abstract}

Keywords: Elderly; Chronic diseases; aging.

\footnotetext{
${ }^{1}$ Curso de Enfermagem pela Faculdade Independente do Nordeste - FAINOR.

${ }^{2}$ Graduação em Ciências Biológicas pela Universidade do Estado do Rio de Janeiro. Mestrado em Ciências pela Universidade do Estado do Rio de Janeiro e Doutorado em Ciências pela Universidade do Estado do Rio de Janeiro;

${ }^{3}$ Graduação em Fisioterapia pela Universidade Estadual do Sudoeste da Bahia, Mestrado em Ciências da Saúde pela Universidade Federal do Rio Grande do Norte, Doutorado em Ciências da Saúde pela Universidade Federal do Rio Grande do Norte e Pós-Doutorado em Saúde Coletiva pelo Instituto de saúde Coletiva da Universidade Federal da Bahia. Atualmente é Gerente de Pesquisa e Formação Continuada da Faculdade Independente do Nordeste;

4 Mestre e Doutoranda do Programa de Pós-graduação Stricto Sensu em Memória:Linguagem e Sociedade da Universidade Estadual do Sudoeste da Bahia. Foi Bolsista em nível de Mestrado Acadêmico pela Fundação de Amparo à Pesquisa do Estado da Bahia - FAPESB. Bacharel em Enfermagem pela Faculdade Independente do Nordeste e Licenciada em Geografia pela Universidade Estadual do Sudoeste da Bahia.
} 


\section{Introdução}

O aumento rápido da população idosa no Brasil e a longevidade é um fato real. Porém, esta longevidade vem na maioria das vezes, associadas às Doenças Crônicas Não Transmissíveis (DCNT), pois essas patologias têm uma relação direta com o envelhecimento (SILVA et al., 2015). Vale lembrar que DCNT são as doenças que mais causam limitações, baixa produtividade, impacto na funcionalidade, redução na qualidade de vida e ainda podem ocasionar morte prematura (SILVA, 2015; LIMA; VALENÇA; REIS, 2016).

As DCNT são patologias multifatoriais que progridem durante a vida, sendo caracterizada como um sério problema de saúde pública. Segundo Organização Mundial de Saúde essas doenças foram causa de $63 \%$ das mortes no mundo e de $72,6 \%$ das mortes no Brasil, em 2013, o que causa impacto na qualidade de vida da população acometida (SIM, 2015).

Dentre os principais fatores de risco para a morbi/mortalidadedas das DCNT estão o tabaco, a má alimentação, o sedentarismo, a hiperglicemia, os níveis alterados de pressão arterial e a obesidade (OLIVEIRA-CAMPOS, 2013). Estes fatores de risco têm como consequência o desenvolvimento de patologias crônicas, como: cardiovasculares, hipertensão, diabetes mellitus e a obesidade. Sendo estas as principais DCNT que acometem a população brasileira, especialmente os idosos (BRASIL, 2014).

Nesse sentido, o idoso portador de uma ou mais DCNT tem mais chances de desenvolver dependência funcional, ter a autonomia reduzida, bem como vivenciar os últimos anos de vida com diversas complicações (FERREIRA et al., 2012).

Baseado nesta rápida exposição pode-se dizer que na faixa etária de idosos é essencial compreender o padrão de desenvolvimento e as consequências das DCNT, pois é por meio de pesquisas deste tipo que será reforçada a atuação dos profissionais de saúde, com destaque para os enfermeiros, especialmente no que diz respeito à prevenção, tratamento e acompanhamento de idosos, de forma a reduzir a morbimortalidade por estas doenças.

Vale salientar ainda que, apesar da realidade brasileira frente às DCNT, sendo elas as patologias que mais apresentam morbimortalidade, estudos atuais são escassos, o que demonstra uma necessidade urgente de realizar pesquisas.

Diante disso, este estudo busca verificar a prevalência das Doenças Crônicas Não Transmissíveis em idosos de uma cidade do interior da Bahia, com o intuito primeiro de 
averiguar a realidade, para favorecer posteriormente estudos mais aprofundados, no que se refere às DCNT que mais foram evidenciadas.

Nesse sentido, este estudo objetiva identificar a prevalência de doenças crônicas em idosos de uma cidade do interior da Bahia.

\section{Materiais e Métodos}

A pesquisa se classifica como descritiva e exploratória, uma vez que faz uso da descrição, coleta e registro de dados. Conforme Gil (2012), a pesquisa exploratória visa proporcionar uma visão geral sobre determinado fato e permite ao pesquisador uma maior familiaridade com o fenômeno que está sendo investigado. Tal tipo de estudo pode ser realizado por meio de distintas técnicas de coleta de dados e informações.

Por outro lado a pesquisa descritiva "Descreve as características de uma determinada população ou um determinado fenômeno, e os interpreta. Não busca interferir e nem modificar a realidade estudada" (GIL, 2012, p. 48).

Em relação à sua natureza, a presente pesquisa se classifica como quantitativa. Este tipo de pesquisa considera que tudo pode ser quantificável. Dessa forma, o investigador traduz em números as opiniões e informações colhidas, analisa os dados e, a partir daí, chega a uma conclusão (MARCONI; LAKATOS, 2011).

O presente estudo foi realizado em uma cidade do interior baiano pertencente à Região Sudoeste.

Os participantes do estudo foram 173 idosos de 60 anos ou mais de ambos os sexos, de um município do interior da Bahia que tem condições mentais para responder aos questionários e que aceitaram participar do estudo.

O cálculo do tamanho amostral foi realizado com um nível de confiança de 95\%, com margem de erro de 5\%. A seleção da amostra foi realizada por meio de amostragem aleatória.

A coleta de dados foi realizada em Grupo de Convivência, na Atenção Básica e Unidade Hospitalar e Escolas, durante os meses de julho e dezembro de 2016.

Foram aplicados dois instrumentos de pesquisa: o Mini Exame do Estado Mental MEEM e Ficha de doenças pré-existentes - critérios ABCDE.

Para avaliação do estado mental foi aplicado o Mini-exame do Estado Mental de Pfeiffer (FOLSTEIN; FOLSTEIN; MCHUGH, 1975), composto por dez perguntas, o qual avalia quatro 
parâmetros: memória a curto e longo prazo; orientação; informações do cotidiano; e capacidade de cálculo. A pontuação seguiu os seguinte critérios: 30 a 26 pontos (funções cognitivas preservadas); 26 a 24 pontos (alteração não sugestiva de déficit) e 23 pontos ou menos (sugestivo de déficit cognitivo). Esse Mini-exame do estado mental possibilitou a exclusão dos idosos que não apresentavam cognição para responder às perguntas da entrevista. Portanto, todos os idosos inseridos no estudo tiveram pontuação a cima de 24 pontos.

O questionário Sóciodemográfico e econômico abrangeu dados relacionados à: idade, sexo, cor, escolaridade, profissão, estado civil, renda e moradia. O mesmo foi utilizado neste estudo apenas para caracterizar o perfil dos idosos participantes do estudo.

A Ficha de doenças pré-existentes - critérios ABCDE é composto de dez variáveis de perguntas, porém para este estudo utilizamos apenas a variável com perguntas referentes à doenças pré-existentes.

Primeiramente o projeto foi encaminhado às instituições que se pretendia realizar o estudo, após a liberação do campo, o projeto foi submetido ao Comitê de Ética em Pesquisa da Faculdade Independente do Nordeste, por meio da Plataforma Brasil. Após a aprovação do estudo pelo comitê de ética, foi dada início à coleta de dados. Os questionários foram aplicados pelos pesquisadores, em locais adequados, com utilização de computadores conectados a internet, pois todos os questionários eram digitais. Após a coleta dos dados foi construído o banco de dados e posteriormente foi realizada a análise descritiva e a confrontação com a literatura atual.

A análise ocorreu por meio da distribuição de frequência absoluta e relativa para as variáveis categóricas e medidas de tendência central (média, mediana e moda) e dispersão (desvio-padrão e amplitude), para as variáveis contínuas. Em todas as análises foram definidos valores de confiança de 5\% $(\alpha=0,05)$. Para a elaboração do banco de dados foi utilizado o aplicativo EPIDATA e, para a análise dos dados, o programa estatístico SPSS $®$, versão 22.0.

Todos os aspectos éticos e legais da Resolução 466/12 do Ministério da Saúde (BRASIL, 2013) foram seguidos rigorosamente. O projeto foi aprovado pelo Comitê de Ética em Pesquisa da Faculdade Independente do Nordeste com parecer de aprovação $n^{\circ}$ 1.670.007. 


\section{Resultados}

Participaram do estudo 173 idosos, sendo que destes 54,91\% (95) são do sexo feminino e $45,09 \%$ (78) do sexo masculino.

Conforme o inventário $\mathrm{ABCDE}$ os idosos eram questionados a cerca do diagnóstico de DCNT. Neste estudo as únicas DCNT referidas por eles foram: depressão; diabetes, cardiopatias, doença renal, câncer e hipertensão.

A Tabela 1 apresenta a distribuição de idosos quanto a doenças crônicas não transmissíveis auto-referida. Na População Geral (PG) $1,15 \%$ (2) auto-referiram ser diagnosticado com depressão, 17,92\% (31) diabetes, 4,62\% (8) cardiopatia, 2,89\% (5) doença renal, 2,89 (5\%) câncer, 98,64\% (171) hipertensão.

No que se refere à População Feminina (PF) 1,15\% (2) auto-referiram ser diagnosticado com a depressão, 15,02\% (26) diabetes, 2,89 (5) cardiopatia, 2,89 (5) doença renal, 0,58\% (1) câncer, 71,68\% (124) hipertensão.

Quanto a População Masculina (PM) as únicas doenças auto-referidas foram: 2,90\% (5) diabetes, 1,73 (3) cardiopatia, 2,31 (4) câncer, 27,16\% (47) hipertensão.

Tabela 1 - Distribuição de idosos quanto a doenças crônicas não transmissíveis auto-referida em uma cidade do interior da Bahia

\begin{tabular}{lcccccc}
\hline \multicolumn{1}{c}{ Variáveis } & \multicolumn{2}{c}{ PG } & \multicolumn{2}{c}{ PF } & \multicolumn{2}{c}{ PM } \\
\hline & $\mathrm{N}$ & $\%$ & $\mathrm{~N}$ & $\%$ & $\mathrm{~N}$ & $\%$ \\
Depressão & 2 & 1,15 & 2 & 1,15 & 0 & 0 \\
Diabetes & 31 & 17,92 & 26 & 15,02 & 5 & 2,90 \\
Cardiopatia & 8 & 4,62 & 5 & 2,89 & 3 & 1,73 \\
Doença & 5 & 2,89 & 5 & 2,89 & 0 & 0 \\
Renal & & & & & & \\
Câncer & 5 & 2,89 & 1 & 0,58 & 4 & 2,31 \\
Hipertensão & 171 & 98,84 & 124 & 71,68 & 47 & 27,16
\end{tabular}

Fonte: Dados da pesquisa 


\section{Discussão}

É sabido que a tendência das DCNTs é crescente, e nos últimos anos diversos fatores contribuem para este crescimento, entre eles está o maior envelhecimento populacional, estilo de vida sedentário, maior exposição aos fatores de risco, aumento da urbanização e industrialização (MALTA; SILVA JR,2013).

No presente estudo sete DCNT's foram auto-referidas pelos idosos, a saber: depressão, diabetes, cardiopatia, doenças renais, câncer e hipertensão. Porém destas, a hipertensão e o diabetes apresentaram um maior índice, sendo que o câncer apresentou um destaque pelo fato de ter sido na maioria dos casos em homens.

O estudo de Andrade et al. (2014), traz dados semelhantes acerca destas doenças crônicas, especialmente a HAS que devido a sua alta prevalência, ainda hoje, consiste em um grave problema de saúde pública. O que pode ser comprovado por meio dos números, pois cerca de $60 \%$ da população idosa brasileira é portadora de HAS, sendo que este o panorama piora na faixa etária >80 anos, chegando a 66\% de prevalência (RAMOS, et al, 2016).

O estudo de Schmidt et al. (2014), também mostra que "a prevalência autorrelatada" de HAS cresceu significativamente e "tem aumentando aproximadamente $0,5 \%$ ao ano", associado ao aumento acentuado, o autor traz que o controle pressórico dos hipertensos tem sido insatisfatório.

Silva et al., (2013) salienta que uma forma eficaz para incentivar o hipertenso no controle pressórico, é facilitar a adesão por meio de um bom acolhimento, com escuta eficaz, bem como atitudes resolutivas e práticas humanizadas, com intuito de aproximar o indivíduo a unidade de saúde e assim ter um aliado na prevenção de danos.

Em relação ao Diabetes Mellitus, a tendência é similar à HAS, pois o índice de DM tem aumentado significativamente na última década. A Sociedade Brasileira de Diabetes estima que hoje exista cerca de 387 milhões de indivíduos com DM e que alcance 471 milhões em 2035.

A prevalência de diabetes em mulheres no presente estudo foi superior aos homens, resultado que foi também confirmado no estudo de Iseret al. (2015). Porém, o que se tem visto é que os homens têm uma maior dificuldade de manter um estilo de vida e um autocuidado condizente com o DM, em comparação as mulheres, o que acaba gerando um índice maior de complicações evitáveis (ROSSANEIS, 2016).

É importante pontuar que o aumento na prevalência dos casos de DM traz impactos negativos e diretos na saúde da população portadora da patologia e impactos na sociedade e 
serviços públicos, uma vez que os gastos onerados pelo Sistema Único de Saúde no que tange ao tratamento do DM é extremamente alto, principalmente na faixa etária de 60 anos ou mais (GRZELCZAK, et al, 2017).

Do mesmo modo, a associação da Hipertensão e Diabetes traz diversos riscos para a saúde dos pacientes. O Diabetes Mellitus tipo 2 quando associado a outras doenças como HAS, displipidemias e obesidade, eleva significativamente a morbidade por desordens cardiovasculares. Nesse sentido, é importante que haja contínuo cuidado destes pacientes, haja vista os altos índices das patologias e os fatores de risco associados (PINHO et al., 2015).

Os dados deste estudo também mostraram que as taxas de Câncer são maiores na população masculina $(2,31 \%)$ em relação à feminina $(0,58 \%)$. Destaca-se principalmente o aumento nos casos de câncer de próstata. Um estudo realizado por Campolina et al (2013) traz dados condizentes com os achados neste estudo e ainda mostram que há aumento na mortalidade por câncer em homens, principalmente no que se refere ao câncer de pulmão, próstata e colorretal.

Neste estudo o câncer em homens chamou atenção pelo fato de ter uma maior prevalência em comparação com as mulheres. De acordo o Instituto Nacional do Câncer (INCA) o câncer de próstata é o segundo mais comum entre os homens, e fica atrás somente do câncer de pele não-melanoma. O INCA ainda traz que a estimativa de novos casos no ano de 2016 eram de 61.200, e a mortalidade em 2013 foi de 13.772 casos de acordo dados do Sistema de Informação de Mortalidade (INCA, 2017).

Diferentemente da realidade do presente estudo, atualmente a incidência de Câncer entre homens e mulheres está distribuída de forma homogênea, haja vista o aumento nos números de casos de câncer de próstata (estimativa de 61 mil casos em 2016), equivalentes aos casos de câncer mama (estimativa de 57 mil casos em 2016) (INCA 2017).

É importante citar que as Doenças Cardiovasculares (DCV) foram auto-referidas pela população geral, com 4,62\% de acometimento e as doenças renais com quase 3\% (2,89\%) de acometimento da população. Ressalta-se que estas doenças podem acometer os idosos isoladamente, ou como complicações decorrentes da HAS e DM (MALTA, 2013, DOS SANTOS e MOREIRA, 2012).

Um estudo publicado por um grupo de especialistas de diversas áreas traz que a mortalidade devido às complicações de DCV é extremamente alta, especialmente em pacientes que possuem a síndrome metabólica, quadro clínico decorrente de diversas alterações como: 
dislipidemias, obesidade e HAS, insuficientemente diagnosticado, e decorrente principalmente da resistência da ação da insulina presente no DM tipo 2 (LÓPEZ-JARAMILLO et al, 2014).

Quanto às Doenças Renais (DR), estas podem está associadas tanto à HAS, como o DM (SANTOS, 2012). A DR está presente em 1,9\% da população idosa com HAS e DM e aparecem como complicações das duas patologias, especialmente da HAS (SANTOS e MOREIRA 2012).

O presente estudo constatou-se que a prevalência de doenças auto-referidas foi maior na população feminina quando comparada à população masculina, o que não representa que os homens adoecem menos, pelo contrário, pois, a mortalidade da população masculina em relação à população feminina desmistifica o estereótipo de que homens adoecem menos quando comparado às mulheres. No entanto, verifica-se que o homem procura menos os serviços de saúde (ALVES et al., 2011).

A menor procura de homens aos serviços de saúde pode ser justificada, pois ainda permanece na cultura brasileira marcas de identidade estereotipadas, de que o homem é o provedor do lar, dominador e para tal, não deve adoecer, por outro lado, a lógica do cuidado é sempre atribuída à mulher (LEVORATO et al., 2014).

\section{Conclusão}

Constatou-se que o perfil de morbidades crônicas encontrados na população pesquisada é semelhante ao perfil em idosos no Brasil. Observa-se tendência crescente especialmente na Hipertensão Arterial Sistêmica, Diabetes Mellitus e câncer na população masculina. O estudo demonstrou ainda um quantitativo maior de doenças auto-referidas por mulheres.

O estudo apresentou algumas limitações como: não associar as doenças autorreferidas com os fatores de risco de cada paciente. Assim, espera-se que mais estudos nestas temáticas possam ser realizados, principalmente a nível populacional da Bahia, pois apesar de possuir grande relevância para a área acadêmica e para a saúde pública, não existe um estudo nesta dimensão.

O que chama atenção neste este estudo é a associação de inúmeras doenças crônicas, o que torna ainda mais complexo a realidade, pois torna as condutas terapêuticas e a assistência mais complexa. Por este fato, acredita-se que este estudo pode contribuir para o entendimento e tratamento da população idosa estudada e possibilitar ações de prevenção voltados a esta população. 


\section{Referências}

LIMA, Pollyanna Viana; VALENÇA, Tatiane Dias Casemiro; REIS, Luciana Araújo dos. Envelhecer com dependência funcional: construindo estratégias de enfrentamento

RevPesq Saúde, 17(2): 96-101, mai-ago, 2016. Disponível em: http://www.periodicoseletronicos.ufma.br/index.php/revistahuufma/article/view/6082

DELGADO, L. A. Avaliação da aptidão física: projeto de elaboração de sistemas de informação. Universidade Federal do Maranhão, São Luís, p. 1-75, 2004.

FERREIRA, Olívia Galvão Lucena; MACIEL, Silvana Carneiro; COSTA, Sônia Maria Gusmão; SILVA, Antonia Oliveira; MOREIRA, Maria Adelaide Silva P.Active agingand its relationshiptofunctionalindependence. TextContextNursing, Florianópolis, 2012 Jul-Sep; 21(3): 513-8. Disponível em: http://www.scielo.br/pdf/tce/v21n3/en_v21n3a04.pdf.

DA SILVA, João Victor Farias, et al. "A relação entre o envelhecimento populacional e as doenças crônicas não transmissíveis: sério desafio de saúde pública." Caderno de GraduaçãoCiências Biológicas e da Saúde-UNIT-ALAGOAS 2.3 (2015): 91-100. Disponível em https://periodicos.set.edu.br/index.php/fitsbiosaude/article/viewFile/2079/1268

MALTA, D. C., SANTOS, M. A. S., ANDRADE, S. S. C. A., OLIVEIRA, T. P., STOPA, S. R., OLIVEIRA, M. M., \& JAIME, P. (2016). Tendência temporal dos indicadores de excesso de peso em adultos nas capitais brasileiras, 2006-2013. Ciênc Saúde Coletiva, 21, 1061-1069. Disponível em

http://www.scielosp.org/scielo.php?script=sci_arttext\&pid=S1413-

$81232016000401061 \&$ lang $=\mathrm{pt}$

OLIVEIRA-CAMPOS, M., RODRIGUES-NETO, J. F., SILVEIRA, M. F., NEVES, D. M. R., VILHENA, J. M., OLIVEIRA, J. F., ...\& DRUMOND, D. (2013). Impacto dos fatores de risco para doenças crônicas não transmissíveis na qualidade de vida. CienSaudeColet, 18(3), 873882. Disponível em

http://www.scielo.br/scielo.php?script=sci_arttext\&pid=S1413-

$81232013000300033 \&$ lang=pt

PORTAL SAÚDE,VIGILÂNCIA DAS DOENÇAS CRÔNICAS NÃO TRANSMISSÍVEIS, 2014, disponível emhttp://portalsaude.saude.gov.br/index.php/o-ministerio/principal/leiamais-o-ministerio/671-secretaria-svs/vigilancia-de-a-a-z/doencas-cronicas-nao-

transmissiveis/14125-vigilancia-das-doencas-cronicas-nao-transmissiveis

ALVES, Railda Fernandes; SILVA, Renata Pimentel; ERNESTO,Monalisa Vasconcelos;LIMA, Ana GabriellaBarros; SOUZA, Fabiana Maria. Gênero e saúde: o cuidar do homem em debate. Psicologia: Teoria e Prática - 2011, 13(3):152-166

LEVORATO, Cleice Daiana; DE MELLO, Luane Marques; DA SILVA, Anderson Soares; NUNES, Altacílio Aparecido. Fatores associados à procura por serviços de saúdenuma perspectiva relacional de gênero. Ciência \& Saúde Coletiva, 19(4):1263-1274, 2014.Doi: 10.1590/1413-81232014194.01242013 
DE ANDRADE, Aluísio Oliveira; DE AGUIAR, Maria Isis Freire; DE ALMEIDA, Paulo César; CHAVES, Emília Soares; ARAÚJO, NeriaVeanne Sousa Silva; NETO, José Borba de Freitas. Prevalência da hipertensão arterial eFatores associados em idosos. RevBrasPromoç Saúde, Fortaleza, 27(3): 303-311, jul./set., 2014

RAMOS, Luiz Roberto. Polifarmácia e polimorbidade em idosos no Brasil: um desafio em saúde pública. Rev Saúde Pública 2016;50(supl 2):9s

SCHMIDT, Maria Inês; DUNCAN, Bruce Bartholow; E SILVA, Gulnar Azevedo; MENEZES, Ana Maria; MONTEIRO, Carlos Augusto; BARRETO, Sandhi Maria; CHOR, Dora; MENEZES, Paulo Rossi. Doenças crônicas não transmissíveis no Brasil: carga e desafios atuais. Series. www.thelancet.com.

DIRETRIZES DA SOCIEDADE BRASILEIRA DE DIABETES (2015-2016) / Adolfo Milech...[et. al.]; organização José Egidio Paulo de Oliveira, Sérgio Vencio - São Paulo: A.C. Farmacêutica, 2016.

ISER, Betine Pinto Moehleckeet al. Prevalência de diabetes autorreferido no Brasil: resultados da Pesquisa Nacional de Saúde 2013. Epidemiol. Serv. Saúde, 305 Brasília, 24(2): 305-314, abr-jun 2015.

GRZELCZAK, Marcos Tadeu; et al. O diabetes sob o aspecto epidemiológico e políticas de promoção de saúde por meio da atividade física. rev cientifica UMC, Mogi das Cruzes, v. 2, n. 2, 2017.

CAMPOLINA, Alessandro Gonçalves; ADAMI, Fernando; SANTOS, Jair Licio Ferreira; LEBRÃOMaria Lúcia. A transição de saúde e as mudanças na expectativa de vida saudável da população idosa: possíveis impactos da prevenção de doenças crônicas. Cad. Saúde Pública, Rio de Janeiro, 29(6):1217-1229, jun, 2013.

INSTITUTO NACIONAL DO CÂNCER (INCA). Próstata.Disponível em: http://www2.inca.gov.br/wps/wcm/connect/tiposdecancer/site/home/prostata acesso em: 13/08/2017.

INSTITUTO NACIONAL DO CÂNCER (INCA). Incidência de Câncer no Brasil. Síntese de Resultados e Comentários. Disponível em: http://www.inca.gov.br/estimativa/2016/sintesede-resultados-comentarios.aspacesso em: 29/08/2017.

MALTA, Deborah Carvalho; SILVA JR, Jarbas Barbosa da. O Plano de Ações Estratégicas para o Enfrentamento das Doenças Crônicas Não Transmissíveis no Brasil e a definição das metas globais para o enfrentamento dessas doenças até 2025: uma revisão. Epidemiol. Serv. Saúde, Brasília, 22(1):151-164, jan-mar 2013.

PINHO, Lucineia, AGUIAR Ana Paula Soares, OLIVEIRA Maíra Rodrigues, BARRETO Nair Amélia Prates, FERREIRA Cristiane Maria Mendes. Hipertensão e dislipidemia em pacientes diabetes mellitus tipo 2: uma revisão integrativa. Revista Norte Mineira de Enfermagem. 2015;4(1):87- 10188 
Mariana Angela Rossaneis2 Maria do Carmo Fernandez Lourenço Haddad3 Thaís Aidar de Freitas Mathias4 Sonia Silva Marcon4 . Diferenças entre mulheres e homens diabéticos no autocuidado com os pés e estilo de vida. Rev. Latino-Am. Enfermagem 2016; 24:e2761. Disponível em: http://www.scielo.br/pdf/rlae/v24/pt_0104-1169-rlae-24-02761.pdf

SILVA Christiana Souto, PAESNeir Antunes, FIGUEIREDOTânia Maria Ribeiro Monteiro de, CARDOSO Maria Aparecida Alves, DA SILVA Ana Tereza Medeiros Cavalcanti, ARAÚJO deJuliana Sousa Soares. Controle pressórico e adesão/vínculo em hipertensos usuários da Atenção Primária à Saúde. RevEscEnferm USP 2013; 47(3):584-90. Disponível em: http://www.scielo.br/pdf/reeusp/v47n3/0080-6234-reeusp-47-3-00584.pdf

LÓPEZ-JARAMILLO Patricio, et al. Consenso latino-americano de hipertensão em pacientes com diabetes tipo 2 e síndrome metabólica. ArqBrasEndocrinolMetab. 2014;58/3. Disponível em: http://www.scielo.br/pdf/abem/v58n3/0004-2730-abem-58-3-0205.pdf

DOS SANTOS, Jênifa C., MOREIRA Thereza Maria Magalhães. Fatores de risco e complicações em hipertensos/diabéticos de uma regional sanitária do nordeste brasileiro. Rev. esc. enferm. USP vol.46 no.5 São Paulo Oct. 2012. Disponível em:http://www.scielo.br/scielo.php?pid=S008062342012000500013\&script=sci_arttext\&tlng $=\mathrm{es}$

\section{Como citar este artigo (Formato ABNT):}

ABREU, Sanmille S.S. de; OLIVEIRA, Andreza G. de; MACEDO, Maria Aparecida S.S.; DUARTE, Stênio F.P.; REIS, Luciana A. dos; LIMA, Pollyanna V. Prevalência de Doenças Crônicas não Transmissíveis em Idosos de uma Cidade do Interior da Bahia. Id on Line Revista Multidisciplinar e de Psicologia, 2017, vol.11, n.38, p.652-662. ISSN: 1981-1179.

Recebido: 30.10 .2017

Aceito: 07.11.2017 